\title{
Numerical modeling of heat transfer in the fuel oil storage tank at thermal power plant
}

\author{
Svetlana A. Kuznetsova \\ Institute of Power Engineering, Tomsk Polytechnic University, Tomsk 634050, Russia
}

\begin{abstract}
Presents results of mathematical modeling of convection of a viscous incompressible fluid in a rectangular cavity with conducting walls of finite thickness in the presence of a local source of heat in the bottom of the field in terms of convective heat exchange with the environment. A mathematical model is formulated in terms of dimensionless variables "stream function - vorticity vector speed - temperature" in the Cartesian coordinate system. As the results show the distributions of hydrodynamic parameters and temperatures using different boundary conditions on the local heat source.
\end{abstract}

Oil is used by more than 200 thermal power plants (TPP) in Russia as a primary or backup fuel [1]. The main problem of its use - is ensuring uninterrupted supply of boilers by heated and filtered oil in the winter. Thus on heating oil during its preparation for burned (in order to reduce viscosity is exponentially dependent on the temperature [1]) is expended considerable part of the generated electricity of TPP. Reducing these costs is one of the important tasks. Its solution is possible at optimal thermal conditions in fuel oil storage and systems supply that fuel.

Choice of thermal conditions empirically possible only at great expense of time and material resources. Therefore, one of the most promising ways to solve this problem is the mathematical modeling of heat transfer processes in the fuel oil storage tank, taking into account conduction, convection, and the heat sink to the environment. So far, conjugate heat transfer problem in fuel oil storage tank is not resolved. Famous models that are used in evaluating the temperature of fuel oil in storage [1], are either empirical or balanced. Last provide an estimate only average in terms of temperatures. In actual practice, due to the high viscosity and low thermal conductivity of fuel oil temperature gradients in all spatial coordinates can be considerable. Therefore the description of the temperature fields in fuel oil storage with using systems of no stationary differential equations is promising.

The aim of work is the mathematical modeling of temperature fields of fuel oil in terms of its storage, including the processes of heat conduction and natural convection with local heat source in the fuel oil storage tank.

As object of research is considered quite typical rectangular tank with a thermally conductive steel walls. Fuel oil is considered as viscous heat-conducting incompressible fluid. The possible presence of water in fuel volume is not considered. There is a source of heat with a constant temperature at the bottom of the tank (Fig. 1). The initial temperature of the liquid fuel exceeds the initial temperature

This is an Open Access article distributed under the terms of the Creative Commons Attribution License 4.0, which permits unrestricted use, distribution, and reproduction in any medium, provided the original work is properly cited. 


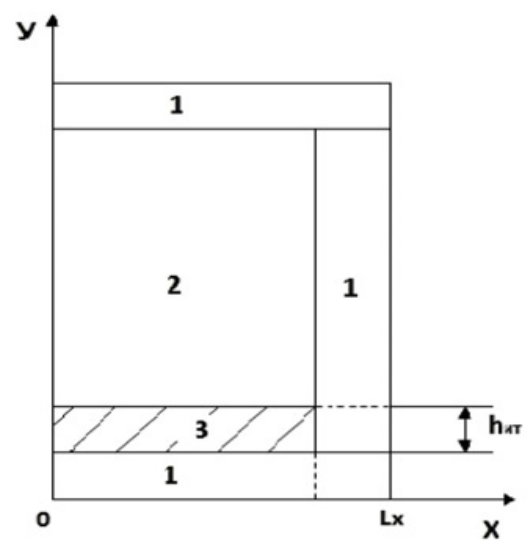

Figure 1. Area of solution the problem.

of the tank wall and the environment. It is assumed that at the boundaries of "liquid - solid wall" the conditions of perfect contact, and on the external borders the heat dissipation into the environment.

Tasks in a similar setting solved for ambient air in a closed cavity with a local source of energy $[2,3]$.

The investigated process is described by analogy with $[2,3]$ the Navier-Stokes equations and the energy equation for the fuel oil and the heat equation for the steel walls of the tank.

The system of equations:

$$
\begin{gathered}
\frac{\partial \Omega}{\partial \tau}+U \frac{\partial \Omega}{\partial X}+V \frac{\partial \Omega}{\partial Y}=\frac{1}{\operatorname{Re}}\left(\frac{\partial^{2} \Omega}{\partial X^{2}}+\frac{\partial^{2} \Omega}{\partial Y^{2}}\right)+\frac{G r}{\operatorname{Re}^{2}}\left(\frac{\partial \Theta}{\partial X}\right), \\
\frac{\partial \Theta}{\partial \tau}+U \frac{\partial \Theta}{\partial X}+V \frac{\partial \Theta}{\partial Y}=\frac{1}{\operatorname{Re} \cdot \operatorname{Pr}}\left(\frac{\partial^{2} \Theta}{\partial X^{2}}+\frac{\partial^{2} \Theta}{\partial Y^{2}}\right) \\
\frac{\partial^{2} \Psi}{\partial X^{2}}+\frac{\partial^{2} \Psi}{\partial Y^{2}}=\Omega \\
\frac{1}{F_{O}} \frac{\partial \Theta}{\partial \tau}=\frac{\partial^{2} \Theta}{\partial X^{2}}+\frac{\partial^{2} \Theta}{\partial Y^{2}}
\end{gathered}
$$

here

$G r=\frac{g \beta L^{3} \Delta T}{v^{2}}-$ Grashof number;

$\beta$ - thermal coefficient of volume expansion; $g$ - acceleration created by the mass forces; $v$ - coefficient of kinematic viscosity of the fluid; Re - Reynolds number; $\mathrm{Pr}$ - Prandtl number; $\mathrm{F}_{0}$ - Fourier number.

Initial conditions: $\Psi(X, Y, 0)=0, \Omega(X, Y, 0)=0, \Theta(X, Y, 0)=0$.

Boundary conditions:

- on the outer contour of considered area

$$
\mid \begin{aligned}
& Y=0, \quad 0 \leq X \leq \frac{l_{1}+l_{2}+l_{u m}}{L} ; \\
& X=\frac{l_{1}+l_{2}+l_{u m}}{L}, \quad 0 \leq Y \leq \frac{h_{1}+h_{2}+H}{L} \\
& Y=\frac{h_{1}+h_{2}+H}{L}, \quad 0 \leq X \leq \frac{l_{1}+l_{2}+l_{u m}}{L}
\end{aligned}
$$




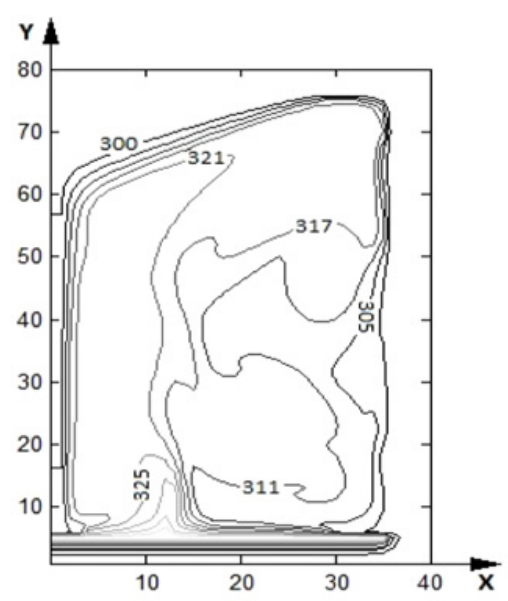

a)

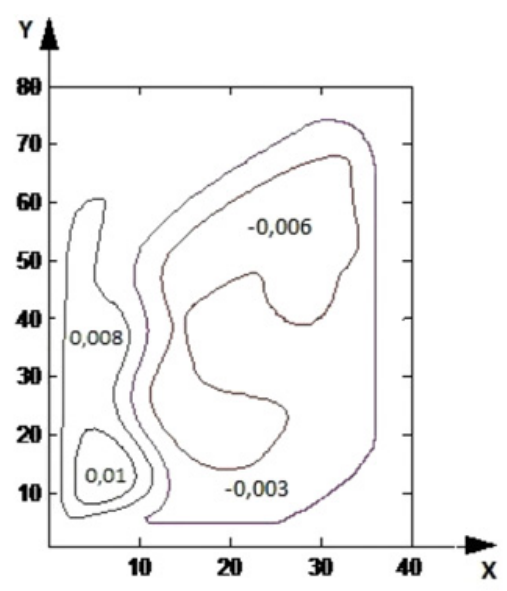

b)

Figure 2. Temperature distribution (a) and contours of the stream function (b) at $\mathrm{T}_{0}=343 \mathrm{~K}$.

are set the boundary conditions of the second kind $\frac{\partial \Theta}{\partial n}=K i$;

- on the axis of symmetry $X=0$ conditions of the form $\frac{\partial \Theta}{\partial X}=\Omega=\Psi=0$;

- at the internal borders between the solid and liquid phase, parallel to the axis $0 \mathrm{Y}$ accepted:

$$
\mid \begin{aligned}
& \Psi=0, \frac{\partial \Psi}{\partial X}=0, \\
& \left\{\begin{array}{l}
\Theta_{w}=\Theta_{f}, \\
\frac{\partial \Theta_{w}}{\partial X}=\lambda_{w, f} \frac{\partial \Theta_{f}}{\partial X},
\end{array} \quad \text { at } X=\frac{l_{1}}{L}, \quad \frac{h_{1}}{L} \leq Y \leq \frac{h_{1}+H}{L}\right.
\end{aligned}
$$

- at the internal borders between the solid and liquid phase, parallel to the axis 0X accepted:

$$
\mid \begin{aligned}
& \Psi=0, \frac{\partial \Psi}{\partial Y}=0, \\
& \left\{\begin{array}{l}
\Theta_{w}=\Theta_{f}, \\
\frac{\partial \Theta_{w}}{\partial Y}=\lambda_{w, f} \frac{\partial \Theta_{f}}{\partial Y},
\end{array} \quad \text { at } Y=\frac{h_{2}}{L}, \quad \frac{l_{1}}{L} \leq X \leq \frac{l_{1}+l_{u m}}{L} ;\right.
\end{aligned}
$$

- On the local heat source are specified boundary conditions of the first kind $\Theta=1$ (Variant 1 ) or the boundary conditions of the second kind $\frac{\partial \Theta}{\partial n}=K i$ (Variant 2)

Here $K i=\frac{q L}{\lambda_{w}\left(T_{i t}-T_{0}\right)}-$ Kirpichev number; $\lambda_{w}$ - coefficient of thermal conductivity of the solid phase; $\lambda_{f}$ - coefficient of thermal conductivity of the liquid phase; $\lambda_{w, f}=\lambda_{w} / \lambda_{f}$ - relative coefficient of thermal conductivity; $q$ - heat flow on the outer boundary solutions.

Formulated above the boundary value problem is solved by finite difference method using the algorithm [4, 5], developed for the numerical solution of nonlinear problems of heat and mass transfer with homogeneous boundary conditions. To substantiate the validity of the results of numerical modeling audited conservatism of used difference scheme by the method developed for solving more complex problems of heat and mass transfer in conditions ignition of liquid fuels with local heating $[6,7]$.

Figures 2-5 shows typical results of numerical investigations as isolines of the stream function and temperature fields. 


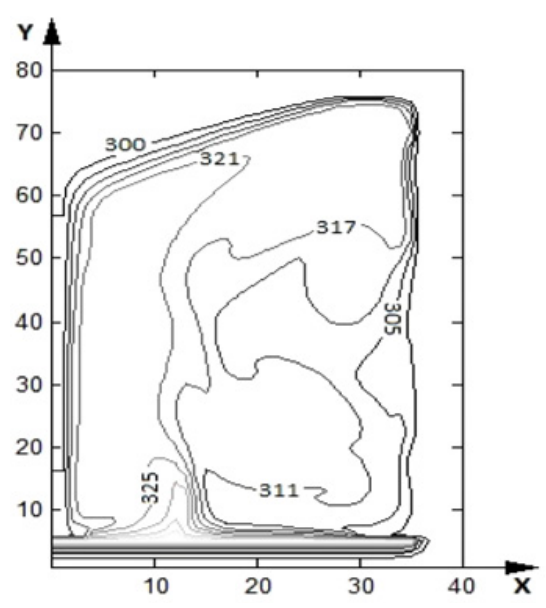

a)

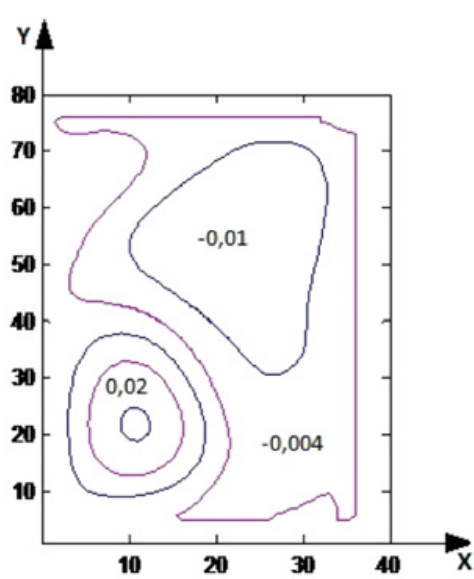

b)

Figure 3. Temperature distribution (a) and contours of the stream function (b) at $\mathrm{T}_{0}=363 \mathrm{~K}$.

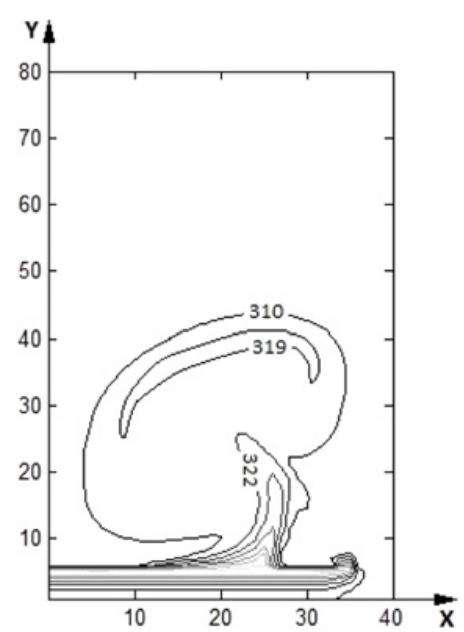

a)

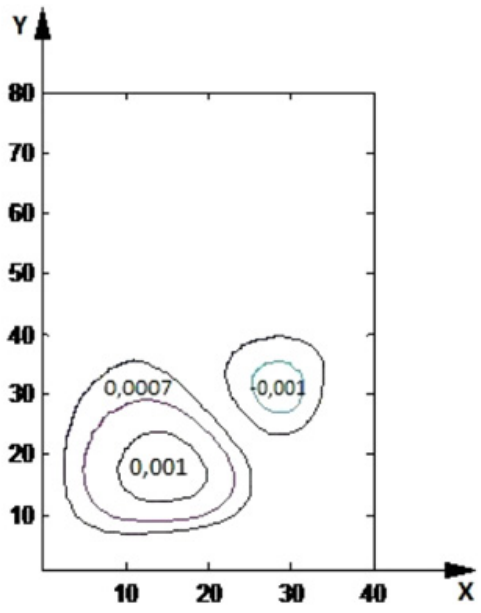

b)

Figure 4. Temperature distribution (a) and contours of the stream function (b) at $\mathrm{q}=200 \mathrm{~W} / \mathrm{m}^{2}$.

Figure 2 clearly seen the formation of several vortices as a result of natural convection in the central part of the reservoir at $\mathrm{T}_{0}=343 \mathrm{~K}$. The character of vortex shedding is due to heat sink on a vertical boundary (the problem is solved in an axisymmetric formulation). The bulk of oil during heating rises near the symmetry axis of the tank and cooled (by heat exchange at the outer boundary of vertical solutions) fuel moves down. In this case clearly shows that, for example, during the time of warm-up about $3600 \mathrm{~s}$ oil temperature field is not homogeneous (there are quite significant temperature gradients).

Increasing the temperature at the bottom boundary of solutions to $363 \mathrm{~K}$ under otherwise appropriate conditions leads to a significant transformation as isolines of the stream function and isotherms due to the intensification of the process of natural convection.

Obtained from the results of numerical modeling of the heat transfer process temperature fields in different heating conditions are grounds for determining the possibility of using previously developed 


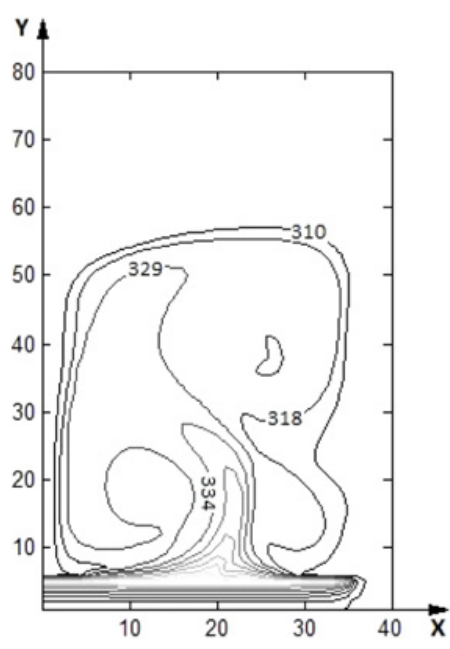

a)

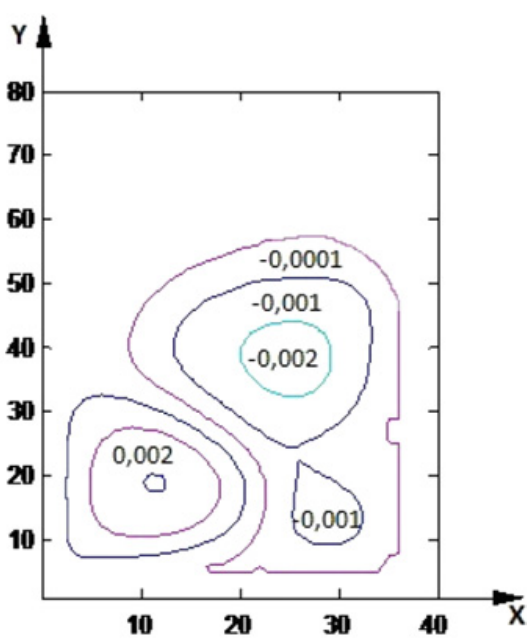

b)

Figure 5. Temperature distribution (a) and contours of the stream function (b) at $\mathrm{q}=500 \mathrm{~W} / \mathrm{m}^{2}$.

models of natural convection [2-4], for the analysis of energy efficiency technologies for regulated heating modes of fuel oil storage.

\section{References}

[1] ECPs and economy of Russia regions. Reference: 7. - Moscow: Energy. (2007)

[2] Kuznetsov G.V., Sheremet M.A. Two-dimensional problem of natural convection in a rectangular domain with local heating and heat-conducting boundaries of finite thickness, Fluid Dynamics. 41. No. 6. (2006) 881-890

[3] Kuznetsov G.V., Sheremet M.A. New approach to the mathematical modeling of thermal regimes for electronic equipment, Russian Microelectronics. 37. No. 2. (2008) 131-138

[4] Kuznetsov G.V., Sheremet M.A. Mathematical modelling of complex heat transfer in a rectangular enclosure, Thermophysics and Aeromechanics. 16. No. 1. (2009) 119-128

[5] Kuznetsov G.V., Strizhak P.A. Heat and mass transfer at the ignition of a liquid substance by a single "hot" particle, Journal of Engineering Thermophysics. 17. No. 3. (2008) 244-252

[6] Kuznetsov G.V., Strizhak P.A. 3D problem of heat and mass transfer at the ignition of a combustible liquid by a heated metal particle, Journal of Engineering Thermophysics. 18. No. 1. (2009) 72-79

[7] Kuznetsov G.V., Strizhak P.A. The influence of heat transfer conditions at the hot particle-liquid fuel interface on the ignition characteristics, Journal of Engineering Thermophysics. 18. No. 2. (2009) 162-167 